\title{
Intrinsic factors associated with medial tibial stress syndrome in athletes: A large case-control study
}

\author{
M Winters, ${ }^{1} \mathrm{MSc} ; \mathbf{H}$ Veldt, ${ }^{2} \mathrm{MD} ; \mathbf{E}$ W Bakker, ${ }^{3,4} \mathrm{PhD} ; \mathbf{M}$ H Moen, ${ }^{5,6} \mathrm{MD}, \mathrm{PhD}$ \\ ${ }^{1}$ University Medical Centre Utrecht, Department of Rehabilitation, Nursing Science and Sport, Utrecht, The Netherlands \\ ${ }^{2}$ Rijnland Medical Centre, Orthopedic Department, Leiderdorp, The Netherlands \\ ${ }^{3}$ Department of Clinical Epidemiology, Biostatistics and Bioinformatics, University of Amsterdam, The Netherlands \\ ${ }^{4}$ KBC Haaglanden, The Hague, The Netherlands \\ ${ }^{5}$ Bergman Clinics, Naarden, The Netherlands \\ ${ }^{6}$ Sports Physician Group, Saint Lucas Andreas Hospital, Amsterdam, The Netherlands
}

Corresponding author: $M$ Winters (marinuswinters@hotmail.com)

\begin{abstract}
Background. Medial tibial stress syndrome (MTSS) is the most common lower-leg injury in athletes, and is thought to be caused by bony overload. To prevent MTSS, both pathophysiological and aetiological factors specific to MTSS need to be identified. The intrinsic risk factors that contribute to the development of MTSS are still uncertain.

Objective. To determine the intrinsic risk factors of MTSS by sampling a large population of athletic MTSS patients and controls.

Methods. Athletes with MTSS and control subjects were medically examined in terms of range of motion of the leg joints (hip abduction, adduction, internal and external range of motion; ankle plantar and dorsal flexion; hallux extension and flexion; subtalar inversion and eversion), measures of over-pronation and maximal calf girth.

Results. Ninety-seven subjects agreed to participate in the study: 48 MTSS patients and 49 active controls. The following variables were considered: gender, age, body mass index (BMI), hip abduction, hip adduction, internal and external hip range of rotation, ankle plantar and dorsal flexion, hallux flexion and extension, subtalar inversion and eversion, maximal calf girth, standing foot angle and navicular drop test. In multivariate logistic regression analysis, hip abduction (odds ratio (OR) $0.82 ; 95 \%$ confidence interval (CI) 0.72 - 0.94 ), ankle plantar flexion (OR 0.73; 95\% CI 0.61 - 0.87) and subtalar inversion (OR 1.24; 95\% CI 1.10 - 1.41) were significantly associated with MTSS. The Nagelkerke $R^{2}$ for this model was 0.76 , indicating that $76 \%$ of the variance in the presence of MTSS could be explained by these variables. Conclusion. Decreased hip abduction, decreased ankle plantar flexion and an increased subtalar inversion could be considered risk factors for MTSS.
\end{abstract}

S Afr J SM 2013;25(3):63-67. DOI:10.7196/SAJSM.376

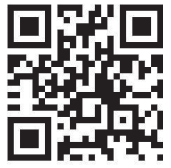

Medial tibial stress syndrome (MTSS) is the most frequently seen overuse injury in the lower leg in jumping and running athletes. ${ }^{[1]}$ Commonly MTSS is defined as 'exercise induced pain on the posteromedial border of the tibia' plus the presence of 'pain on palpation along the posteromedial border over five or more consecutive centimetres.'.[2] Until now, no effective strategies aimed at the prevention of MTSS have been described in athletic populations. ${ }^{[3]}$ For prevention, knowledge of the pathophysiological and aetiological risk factors specific to MTSS is essential.

Regarding pathophysiology, MTSS is thought to be a bony overload injury. ${ }^{[3]}$ The most firm evidence for this is derived from a case control study, which showed that local bone-mineral density was decreased in MTSS patients. A follow-up study concluded that bone-mineral density was restored when patients had recovered, confirming that the bony overload theory was likely. ${ }^{[4]}$

Regarding aetiological factors, several studies have been conducted. However, the intrinsic factors that contribute to the onset of MTSS are still uncertain, since many studies have contradictory results. Female gender, a high body mass index (BMI) and foot pronation were shown to be associated with MTSS in several studies. ${ }^{[3]}$ In addition to these factors, other possible risk factors for MTSS have been studied as well, but mostly in small army populations; ${ }^{[2,5-7]}$ consequently, the results may be not applicable to athletic populations. Furthermore, the results of risk-factor studies are often conflicting. This may be the result of the variety of factors assessed and the small samples studied.

The aim of this study was to determine the intrinsic risk factors for MTSS by sampling a large population of athletic MTSS patients and controls. With a better understanding of the risk factors for MTSS, the prevention of the syndrome by targeting of these risk factors becomes more feasible.

\section{Methods}

Subjects and procedure

Subjects with MTSS were recruited at the Sports Medical Department of the Rijnland Medical Centre when they signed up for treatment. To facilitate patient recruitment, local physiotherapists and general practitioners in the hospital area were informed about the study telephonically and by email. Healthy athletic control subjects (aged $\geq 16$ years) were recruited at the Central Institute of Education for Sports Instructors (CIOS) in Haarlem and the Hague Academy for Physical Education (HALO) in The Hague. One teacher at each location informed 
all first-year students about the study and asked them to participate voluntarily. On average, the athletic controls performed approximately 15 hours of sports activities per week at school (mainly jumping and running activities). The athletic controls were free of MTSS symptoms (leg pain during exercise) upon assessment. Controls were excluded if they were not able to perform physical activity in the 6 months prior to the study's start.

MTSS was defined as 'exercise induced pain along the posteromedial border' plus the presence of 'pain on palpation along the posteromedial border of the tibia over a length of five or more centimetres'. ${ }^{[2]}$ Patients (age $\geq 16$ years) with MTSS complaints persisting for longer than 2 weeks were included in the study when no tibial stress fracture or chronic exertional compartment syndrome (CECS) was suspected based on clinical examination. Pain in tibial stress fractures is often focal, whereas pain in MTSS is more diffuse. Furthermore, in contrast with MTSS, pain in tibial stress fractures is usually characterised by a sudden onset. Patients with CECS usually indicate a burning, cramping pain over the involved compartment. Pain increases with continued exercise and ceases after exercise. ${ }^{[8]}$ Patients with a history of lower-leg or foot fractures and patients who previously had surgery on the legs and feet were excluded.

All participants signed informed consent. The local medical ethics committee approved the study prior to its commencement.

\section{Demographic information and physical examination}

Two sports physicians obtained the participants' demographic information using a standardised form. Gender (male/female), age (years), body weight $(\mathrm{kg})$ and body length $(\mathrm{cm})$ were recorded. The medical examination was conducted according to Moen et al. ${ }^{[5]}$ One sports physician performed the standardised physical examination in the test group with MTSS, whereas the other performed this in the control group. A goniometer was used to assess articular range of motion (Zimmer Ltd., Swindon, UK). This method has a good inter-observer reliability. ${ }^{[9,10]}$ Prior to this examination, several familiarisation sessions were held to minimise inter-observer error. The different parameters were examined as follows:

- Hip abduction and adduction ranges of motion were measured with the patient supine and with the knee extended and the hip in neutral position. The stationary arm was aligned vertically with the body, and the movable arm was aligned with the basis of the patella. The hip was then moved until the pelvis tilted or declined on the ipsilateral side, for hip abduction and adduction, respectively. ${ }^{[11]}$

- Hip internal and external ranges of motion were measured with the patient supine and the knee and hip flexed to $90^{\circ}$. The hip was internally and externally rotated to a firm end feel. Range of motion, relative to the initial position was measured in degrees. ${ }^{[6]}$

- Ankle dorsal and plantar flexion ranges of motion were measured with the subject in the prone position, with the knees extended and the ankles hanging over the edge of the table. The measurement was obtained with the axis of the goniometer on the lateral malleolus. The stationary arm was aligned with the head of the fibula and the movable arm was aligned with the fifth metatarsal. The investigator passively dorsiflexed and plantar flexed the foot until tension was noticed..$^{[9]}$

- Hallux extension and flexion were measured with the goniometer until tension was felt, with the subject in a supine position and the knees extended. The movable arm was aligned with the hallux and the stationary arm was aligned with the first metatarsal bone. ${ }^{[10]}$

- Subtalar eversion and inversion of the ankle were assessed with the subject supine and the knees extended. With the subtalar joint in neutral stance, an inclinometer was placed perpendicular to the foot over the posterior aspect of the calcaneus. Maximal inversion and eversion were measured from this position. ${ }^{[10]}$

- Maximal calf girth was measured (in $\mathrm{cm}$ ) with the subject standing relaxed and upright. A measuring tape was used to obtain the maximum girth of the relaxed calf. ${ }^{[12]}$

- The standing foot angle was measured according to Sommer and Vallentyne. ${ }^{[13]}$ With the subject standing, the angle between the first metatarsal, medial malleolus and the navicular bone was measured. Results were dichotomised to $\geq 140^{\circ}$ and $<140^{\circ}$. This cut-off was used because it had the best sensitivity and specificity $(71 \%$ and $70 \%$, respectively). ${ }^{[13]}$

- The navicular drop test was performed after marking the navicular prominence with the subject sitting in a chair and the feet on the ground (non-weight-bearing) in neutral subtalar position. The distance from the prominence to the floor was then measured. The test was repeated with the subject standing on both feet, shoulderwidth apart (weight-bearing). The two measurements were subtracted, resulting in a difference score (in $\mathrm{cm}$ ). The results were dichotomised $(<0.5 \mathrm{~cm}$ and $>0.5 \mathrm{~cm})$ according to Bennett et al..$^{[7]}$

\section{Data analysis}

Data analysis was performed by two of the investigators (MW and EB) using SPSS version 20.0. Demographic and intrinsic risk factors were presented in terms of means with standard deviations (SDs) for continuous data. In the case of skewed distributions, medians and ranges were presented. Nominal data were presented with their percentages.

Differences between the groups were assessed using Student's $t$-test, or when the assumptions were violated, the Mann-Whitney U-test for continuous variables and chi-square test for nominal data.

Logistic regression was used to assess the association between the dependent (MTSS yes/no) and independent variables. After univariate analysis, a multivariate logistic regression analysis (backward Wald) was conducted on those independent variables that showed a relation to the presence of MTSS. Considering the number of subjects included in this study, we limited the amount of variables in the multivariate model to 9 . Threshold for entry of independent variables in the multivariate model was $p<0.1$ and for removal, $p>0.2$. When more than 9 variables showed a possible relationship with MTSS in univariate analysis, a threshold of $p<0.05$ was used. The Nagelkerke $R^{2}$ was used to assess the explained variance of the model.

\section{Results}

In total, 97 subjects (Table 1) agreed to participate in the study: 48 MTSS patients and 49 active controls. Gender, age, body mass index (BMI), decreased hip abduction, increased hip adduction, increased ankle plantar and dorsal flexion, decreased hallux flexion, increased subtalar inversion and eversion and maximal calf girth were significantly different between the groups (Table 1).

After univariate analysis, 9 variables - gender, age, BMI, hip abduction and adduction, ankle plantar flexion, subtalar inversion 
Table 1. Intrinsic factors and differences between MTSS patients and active controls ${ }^{*}$

\begin{tabular}{|c|c|c|c|}
\hline Risk factors & MTSS group $(N=48)$ & Control group $(N=49)$ & $p$-value \\
\hline Women, $n(\%)$ & $27(56.3)$ & $16(32.7)$ & 0.02 \\
\hline Age in years, mean $( \pm S D)$ & $20.90( \pm 4.34)$ & $18.33( \pm 3.51)$ & $<0.01$ \\
\hline BMI, mean $( \pm S D)$ & $22.39( \pm 2.12)$ & $21.29( \pm 2.12)$ & 0.02 \\
\hline Hip abduction, mean $( \pm$ SD) & $51.15( \pm 7.94)$ & $60.00( \pm 7.00)$ & $<0.01$ \\
\hline Hip adduction, mean $( \pm$ SD) & $35.00( \pm 9.78)$ & $29.49( \pm 3.85)$ & $<0.01$ \\
\hline Hip internal rotation $\left(^{\circ}\right)$, mean $( \pm S D)$ & $45.00( \pm 10.21)$ & $45.71( \pm 5.30)$ & 0.67 \\
\hline Hip external rotation $\left({ }^{\circ}\right)$, mean $( \pm \mathrm{SD})$ & $43.54( \pm 8.31)$ & $43.57( \pm 4.89)$ & 0.98 \\
\hline Ankle dorsal flexion $\left(^{\circ}\right)$, mean $( \pm \mathrm{SD})$ & $20.61( \pm 7.19)$ & $18.59( \pm 4.12)$ & 0.10 \\
\hline Ankle plantar flexion $\left({ }^{\circ}\right)$, mean $( \pm$ SD $)$ & $45.11( \pm 8.66)$ & $53.06( \pm 5.76)$ & $<0.01$ \\
\hline Hallux flexion, mean $( \pm \mathrm{SD})$ & $34.81( \pm 8.26)$ & $37.96( \pm 5.49)$ & 0.03 \\
\hline Hallux extension, mean $( \pm S D)$ & $55.95( \pm 12.81)$ & $57.86( \pm 8.90)$ & 0.41 \\
\hline Subtalar inversion, mean $( \pm$ SD $)$ & $33.44( \pm 8.91)$ & $28.27( \pm 4.95)$ & $<0.01$ \\
\hline Subtalar eversion, mean $( \pm \mathrm{SD})$ & $20.78( \pm 7.68)$ & $18.16( \pm 3.01)$ & 0.03 \\
\hline Maximal calf girth, mean $( \pm \mathrm{SD})$ & $37.14( \pm 2.21)$ & $35.89( \pm 2.17)$ & 0.01 \\
\hline Standing foot angle $>140^{\circ}, n(\%)$ & $26(54.1)$ & $34(69.3)$ & 0.21 \\
\hline Navicular drop test $>0.5 \mathrm{~cm}, n(\%)$ & $25(52.1)$ & $22(44.9)$ & 0.41 \\
\hline
\end{tabular}

Table 2. Univariate and multivariate logistic regression analysis for MTSS risk factors

\begin{tabular}{|c|c|c|c|}
\hline \multirow[b]{2}{*}{ Parameters } & \multicolumn{2}{|c|}{ Univariate logistic regression } & \multirow{2}{*}{$\begin{array}{l}\text { Multivariate logistic regression } \\
\text { OR }(95 \% \mathrm{CI})\end{array}$} \\
\hline & OR (95\% CI) & $p$-value & \\
\hline Gender (female) & $2.65(1.16-6.06)$ & $0.02^{*}$ & $6.11(0.90-41.61)$ \\
\hline Age & $1.21(1.06-1.39)$ & $<0.01^{\star}$ & $0.99(0.89-1.09)$ \\
\hline BMI & $1.28(1.05-1.57)$ & $0.02^{*}$ & $1.39(0.99-1.97)$ \\
\hline Hip abduction & $0.85(0.79-0.91)$ & $<0.01^{*}$ & $0.82(0.72-0.94)^{\dagger}$ \\
\hline Hip adduction & $1.12(1.04-1.20)$ & $<0.01^{*}$ & $1.05(0.92-1.19)$ \\
\hline Hip internal rotation & $0.99(0.94-1.04)$ & 0.66 & - \\
\hline Hip external rotation & $1.00(0.94-1.06)$ & 0.98 & - \\
\hline Ankle dorsal flexion & $1.07(1.00-1.16)$ & 0.11 & - \\
\hline Ankle plantar flexion & $0.85(0.78-0.92)$ & $<0.01^{\star}$ & $0.73(0.61-0.87)^{\dagger}$ \\
\hline Hallux flexion & $0.93(0.87-1.00)$ & 0.04 & - \\
\hline Hallux extension & $0.98(0.95-1.02)$ & 0.40 & - \\
\hline Subtalar inversion & $1.11(1.04-1.19)$ & $<0.01^{*}$ & $1.24(1.10-1.41)^{\dagger}$ \\
\hline Subtalar eversion & $1.10(1.00-1.20)$ & $0.03^{*}$ & $0.99(0.86-1.14)$ \\
\hline Maximal calf girth & $1.30(1.06-1.60$ & $0.01^{*}$ & $1.22(0.74-1.99)$ \\
\hline Standing foot angle $>140^{\circ}$ & $0.56(0.23-1.39)$ & 0.21 & - \\
\hline Navicular drop test & $0.71(0.32-1.61)$ & 0.41 & - \\
\hline \multicolumn{4}{|c|}{$\mathrm{OR}=$ odds ratio; $\mathrm{CI}=$ confidence interval; $\mathrm{BMI}=$ body mass index. } \\
\hline \multicolumn{4}{|c|}{${ }^{*}$ Parameters with a relationship with MTSS after univariate regression analysis and which were entered into the multivariate model $(p<0.1)$. } \\
\hline
\end{tabular}


and eversion, and maximal calf girth - were eligible for selection into the multivariate model $(p<0.1)$.

In the multivariate model, reduced hip abduction, ankle plantar flexion and increased mobility of subtalar inversion were significantly associated with MTSS and could therefore be considered risks factor for MTSS (Table 2).

All parameters were measured on a continuous scale. For hip abduction, the odds ratio (OR) for MTSS decreased by a factor of 0.82 for each additional degree of hip abduction. Consequently, for every additional $5^{\circ}$ difference in range of motion, the odds for MTSS decreased by a factor of $0.37\left(0.82^{5}\right)$. For ankle plantar flexion, an OR of 0.73 was found. For two subjects with a difference of $5^{\circ}$ in plantar flexion of the ankle, the odds for MTSS would be $0.21\left(0.73^{5}\right)$. In contrast, with every additional degree in subtalar inversion, the odds for MTSS increased by a factor of 1.24; therefore, with a $5 \mathrm{~cm}$ increase in subtalar inversion, the odds for MTSS were $2.93\left(1.24^{5}\right)$. The percentage of the total log likelihood for MTSS explained by the significant independent variables (i.e. hip abduction, ankle plantar flexion and subtalar inversion) was $76.7 \%$ (Nagelkerke $R^{2}$ ).

\section{Discussion}

This is the largest study assessing intrinsic factors (gender, age, BMI, hip adduction, hip abduction, hip internal range of motion, hip external range of motion, ankle dorsal flexion, ankle plantar flexion, hallux flexion, hallux extension, subtalar inversion, subtalar eversion, maximal calf girth, standing foot angle, navicular drop test) of MTSS in athletes.

Following multivariate logistic regression analysis, hip abduction, ankle plantar flexion and subtalar inversion were associated with MTSS, explaining $76 \%$ of the variance in the presence of MTSS.

This is the first study to conclude that hip abduction is associated with MTSS. The mean hip abduction was $51^{\circ}$ in athletes with MTSS, and $60^{\circ}$ in athletes without MTSS. Decreased hip abduction is also considered a risk factor for MTSS.

In contrast to previous findings, we did not find differences in hip internal rotation between the groups $(p>0.05)$. The mechanisms through which hip range of motion affect loading on the tibia are unclear. Burne et al. ${ }^{[6]}$ hypothesised that alterations in hip range of motion cause stride patterns that could increase loading of the posteromedial side of the tibia.

We found increased subtalar inversion to be significantly associated with MTSS. This was previously found by Viitasalo and Kvist. ${ }^{[14]}$ In the study by Hubbard et al., ${ }^{[15]}$ no significant difference in subtalar inversion was found between those who developed MTSS and those who did not.

The mean ankle plantar flexion range of motion was $45^{\circ}$ in athletes with MTSS and $53^{\circ}$ in athletes without MTSS. This is in contrast to previous studies that found a higher ankle plantar flexion range of motion to be a risk factor for MTSS. ${ }^{[5,15]}$ Moen et al..$^{[5]}$ speculated that an increased plantar flexion range of motion leads to more forefoot running, leading to more loading on the tibia. Our results oppose this suggestion.

In this study, cases and controls differed significantly in terms of gender and BMI. Gender and BMI were non-significant (both $p=0.06$ ) in the multivariate regression analysis. This supports Hubbard et al. $\mathbf{s}^{[5]}$ conclusion. In contrast, Plisky et al. ${ }^{[16]}$ and Bennett et al. ${ }^{[7]}$ found that women have a higher chance of developing MTSS. Therefore, whether or not gender is a risk factor for MTSS remains unclear.

Various studies have shown that over-pronation is a risk factor for tibial stress injuries. ${ }^{[3]}$ This study, however, suggests that over-pronation during stance is not an important factor in MTSS development $(p>0.05)$. These results concur with the studies by Hubbard et al. ${ }^{[15]}$ and Plisky et al. ${ }^{[16]}$ Future studies are warranted in which athletes with excessive pronation and without complaints are provided with an antipronation inlay with the aim of preventing MTSS.

We chose to assess static instead of dynamic parameters, as it is more practical for a clinician to assess static risk factors in, e.g., an outer clinic. We have completed another study that assessed dynamic parameters (Moen et al., unpublished data 2013); however, the data have not been presented here. In the study, it became apparent that measuring dynamic parameters was time-consuming and difficult to perform, and consequently not practical for clinicians.

\section{Study limitiations}

This study had several limitations. Firstly, we did not measure the volume and intensity of exercise across groups; however, it is unlikely that exercise confounded the relationship between the mentioned intrinsic factors and MTSS. The athletic controls were students who performed approximately 15 hours of running and jumping sports activities per week. We know that the patients endured similar exercise volumes and intensities. Secondly, both groups were examined by two different observers; despite this, the inter-observer reliability was found to be fair for these measurements. Thirdly, the sports physicians were not blinded to whether the subjects were test cases or controls. One of the results could possibly have been the result of chance. We allowed 9 variables in the multivariate model. Some experts advise to impute one parameter for each subject, others for each case. In conclusion, the study results need to be interpreted with caution. Further research focused on intrinsic factors is required before hard conclusions can be drawn.

As part of the prevention of MTSS, trainers, physicians and therapists should assess athletes' hip abduction, ankle plantar flexion and subtalar inversion prior to commencing a training regime, to detect abnormalities that could lead to the onset of MTSS.

\section{Conclusion}

We assessed intrinsic risk factors for MTSS in a large population of athletic MTSS patients, and found that a decreased hip abduction, ankle plantar flexion and an increased subtalar inversion could be considered such.

\section{References}

1. Taunton JE, Ryan MB, Clement DB, et al. A retrospective case-control analysis of 2002 running injuries. Br J Sports Med 2002;36(2):95-101. [http://dx.doi.org/10.1136/ bjsm.36.2.95]

2. Yates $\mathrm{B}$, White $\mathrm{S}$. The incidence and risk factors in the development of medial tibial stress syndrome among naval recruits. Am J Sports Med 2004;32(3):772-780. [http:// dx.doi.org/10.1177/0095399703258776]

3. Moen MH, Tol JL, Weir A, Steunebrink M, de Winter TC. Medial tibial stress syndrome: A critical review. Sports Med 2009:39(7):523-546. [http://dx.doi. org/10.2165/00007256-200939070-00002]

4. Magnusson HI, Ahlborg HG, Karlsson C, Nyquist F, Karlsson MK. Low regional tibial bone density in athletes with medial tibial stress syndrome normalizes after recovery from symptoms. Am J Sports Med 2003;31(4):596-600. 
5. Moen MH, Bongers T, Bakker EW, et al. Risk factors and prognostic indicators for medial tibial stress syndrome. Scan J Med Sci Sports 2012;22(1):34-39. [http://dx.doi. org/10.1111/j.1600-0838.2010.01144.x]

6. Burne SG, Khan KM, Boudville PB, et al. Risk factors associated with exertional tibial pain: A twelve months prospective clinical study. Br J Sports Med 2004:38(4):441-445. [http://dx.doi.org/10.1136/bjsm.2002.004499]

7. Bennett JE, Reinking MF, Pluemer B, et al. Factors contributing to the development of medial tibial stress syndrome in high school runners. J Orthop Sports Phys Ther 2001:31(9):504-510.

8. Edwards PH Jr, Wright ML, Hartman JF. A practical approach for the differential diagnosis of chronic leg pain in the athlete. Am J Sports Med 2005;33(8):1241-1249. [http://dx.doi.org/10.1177/0363546505278305]

9. Jonson SR, Gross MT. Intraexaminer reliability, interexaminer reliability and mean values for nine lower extremity skeletal measures in healthy naval midshipmen. J Orthop Sports Phys Ther 1997:25(4):253-263.

10. Gheluwe van B, Kirby AK, Roosen P, Phillips RD. Reliability and accuracy of biomechanical measurements of the lower extremity. J Am Podiatr Med Assoc 2002:92(6):317-326
11. Prather H, Harris-Hayes M, Hunt DM, Steger-May K, Mathew V, Clohisy JC. Reliability and agreement of hip range of motion and provocative physical examination tests in asymptomatic volunteers. PM\&R 2010;2(10):888-895. [http://dx.doi.org/10.1016/j. pmrj.2010.05.005]

12. Zatsiorsky V, Seluyanov V, Cugunova L. In vivo body segment inertial parameter determination using a gamma-scanner method. In: Berme B, Capozzo A, eds. Biomechanics of Human Motion: Applications in Rehabilitation, Sports and Ergonomics. Worthington, OH: Betec Corporation, 1990:186-202.

13. Sommer HM, Vallentyne SW. Effect of foot posture on the incidence of medial tibial stress syndrome. Med Sci Sports Exerc 1995:27(6):800-804.

14. Viitasalo JT, Kvist M. Some biomechanical aspects of the foot and ankle in athletes with and without shin splints. Am J Sports Med 1989;11(3):125-130. [http://dx.doi. org/10.1177/036354658301100304]

15. Hubbard TJ, Carpenter EM, Cordova ML. Contributing factors to medial tibial stress syndrome: A prospective investigation. Med Sci Sports Exer 2009:41(3):490-496.

16. Plisky MS, Rauh MJ, Heiderscheit B, Underwood FB, Tank RT. Medial tibial stres syndrome in high school cross-country runners: Incidence and risk factors. J Orthop Sports Phys Ther 2007:37(2):40-47. [http://dx.doi.org/10.2519/jospt.2007.2343] 